\title{
PSO-Based Tsallis Thresholding Selection Procedure for Image Segmentation
}

\author{
P.D. Sathya \\ Research Scholar \\ Dept. of Electrical Engineering \\ Annamalai University \\ Chidambaram - 608002 \\ Tamilnadu, INDIA
}

\author{
R. Kayalvizhi \\ Professor \\ Dept. of Instrumentation Engineering \\ Annamalai University \\ Chidambaram - 608002 \\ Tamilnadu, INDIA
}

\begin{abstract}
Multilevel thresholding is a method that is widely used in image segmentation. The thresholding problem is treated as an optimization problem with an objective function. In this article, a simple and histogram based approach is presented for multilevel thresholding in image segmentation. The proposed method combines Tsallis objective function and Particle Swarm Optimization (PSO). The PSO algorithm is used to find the optimal threshold values which maximize the Tsallis objective function. Simulations are performed over various standard test images with different number of thresholds and comparisons are performed with Genetic Algorithm (GA). The experimental results show that the proposed PSO based thresholding method performs better than the GA method.
\end{abstract}

\section{Keywords}

Image thresholding, Tsallis objective function, particle swarm optimization.

\section{INTRODUCTION}

Image segmentation plays an important role in understanding and analyzing image. In particular, region segmentation and object detection in image are both essential procedures for practical applications. Methods for image segmentation [1] include texture analysis based methods, histogram thresholding based methods, clustering based methods and region based split and merging methods, among which thresholding based image segmentation [1-4] is widely used in many applications, such as document processing and object detection, as it is simple and efficient as regards dividing image into the foreground and background.

Thresholding involves bi-level thresholding and multilevel thresholding. Bi-level thresholding classifies the pixels into two groups, one including those pixels with gray levels above certain threshold, the other including the rest. Multilevel thresholding divides the pixels into several classes. The pixels belonging to the same class have gray levels within a specific range defined by several thresholds. Both bi-level and multilevel thresholding methods can be classified into parametric and nonparametric approaches.

In parametric approach, the gray level distribution of each class has a probability density function that is assumed to obey a given distribution. An attempt to find an estimate of the parameters of the distribution that best fits the given histogram data is made by using the least-squares method. It typically leads to a nonlinear optimization problem, of which the solution is computationally expensive and time-consuming.

The nonparametric approach is based on a search of the thresholds optimizing an objective function such as entropy and betweenclass variance method. A great number of thresholding methods belonging to parametric and nonparametric approaches have been proposed in order to perform bi-level thresholding. Of particular interest is an information theoretic approach that based on the concept of entropy introduced by Shannon in information theory [5]. The principle of entropy is to use uncertainty as a measure to describe the information contained in a source. Using maximum entropy as an optimal criterion for image thresholding was first proposed by Pun [6] [7]. It was later corrected and improved by Kapur et al. [3]. The concept was further generalized to Renyi's entropy. Basically, the entropy thresholding considers an image histogram as a probability distribution, and selects as an optimal threshold value that yields the maximum entropy. More specifically, a best entropy thresholded image is the one that preserves as much information as possible that is contained in the original unthresholded image in terms of Shannon's entropy.

Recent developments of statistical mechanics based on a concept of non-extensive entropy, also called Tsallis entropy, have intensified the interest of investigating a possible extension of Shannon's entropy to Information Theory [8]. This interest appears mainly due to similarities between Shannon and Boltzmann/Gibbs entropy functions. The Tsallis entropy is a new proposal in order to generalize the Boltzmann/Gibbs's traditional entropy to non-extensive physical systems.

All the above methods were originally developed for bi-level thresholding. They are extendable to multilevel thresholding as well. In [9], the Otsu's function is modified by a fast recursive algorithm along with a look-up-table for multilevel thresholding. In [10], Lin has proposed a fast thresholding computation using Otsu's function. Another fast multilevel thresholding technique has been proposed by Yin [11]. However, the amount of thresholding computation significantly increases with this extension. To overcome this problem, several techniques have been proposed based on optimization techniques.

Several techniques using genetic algorithms (GAs) have been proposed to solve the multilevel thresholding problem [12], [13]. Though GA-based approaches perform well for complex optimization problems, recent research has identified certain deficiencies [14], particularly for problems in which variables are 
highly correlated. In such cases, the GA crossover and mutation operators do not generate individuals with better fitness of offspring as the chromosomes in the population pool have some structure towards the end of the search.

The PSO, first introduced by Kennedy and Eberhart [15] is a flexible, robust, population based stochastic search/optimization algorithm with inherent parallelism. In recent years this method has gained popularity over its competitors and is increasingly gaining acceptance for solving many image processing problems [16], [17] and [18], due to its simplicity, superior convergence characteristics and high solution quality.

This paper presents Tsallis entropy thresholding method based on particle swarm optimization (PSO) algorithm. In this method a new parameter $\mathrm{q}$ is introduced as a real number associated with the non-extensivity of the system, and it is system dependant. From the simulation results it is verified that the Tsallis based PSO algorithm can efficiently improve the performance in finding optimal threshold values.

\section{MULTILEVEL THRESHOLDING PROBLEM FORMULATION}

In this section, a new thresholding method is proposed based on the entropy concept. This method is similar to the maximum entropy sum method of Kapur et al [3]; however the Tsallis nonextensive entropy concept is used for customizing information theory.

Let there be $\mathrm{L}$ gray levels in a given image and these gray levels are in the range $\{0,1,2, \ldots \ldots \ldots,(\mathrm{L}-1)\}$. Then one can define $\mathrm{P}_{\mathrm{i}}=$ $\mathrm{h}(\mathrm{i}) / \mathrm{N},(0 \leq \mathrm{i} \leq(\mathrm{L}-1))$ where $\mathrm{h}(\mathrm{i})$ denotes number of pixels for the corresponding gray-level $\mathrm{L}$ and $\mathrm{N}$ denotes total number of pixels in the image which is equal to $\sum_{i=0}^{\mathrm{L}-1} \mathrm{~h}(\mathrm{i})$.

Tsallis bi-level thresholding can be described as follows

$$
\mathrm{f}(\mathrm{t})=\operatorname{argmax}\left[\mathrm{S}_{\mathrm{q}}{ }^{\mathrm{A}}(\mathrm{t})+\mathrm{S}_{\mathrm{q}}{ }^{\mathrm{B}}(\mathrm{t})+(1-\mathrm{q}) \cdot \mathrm{S}_{\mathrm{q}}{ }^{\mathrm{A}}(\mathrm{t}) \cdot \mathrm{S}_{\mathrm{q}}{ }^{\mathrm{B}}(\mathrm{t})\right]
$$

where

$\mathrm{q}$ is an entropic index,

$$
\begin{aligned}
& \mathrm{S}_{\mathrm{q}}{ }^{\mathrm{A}}(\mathrm{t})=\frac{1-\sum_{\mathrm{i}=0}^{\mathrm{t}-1}\left(\frac{\mathrm{P}_{\mathrm{i}}}{\mathrm{P}^{\mathrm{A}}}\right)}{\mathrm{q}-1}, \mathrm{P}^{\mathrm{A}}=\sum_{\mathrm{i}=0}^{\mathrm{t}-1} \mathrm{P}_{\mathrm{i}} \\
& \mathrm{S}_{\mathrm{q}}^{\mathrm{B}}(\mathrm{t})=\frac{1-\sum_{\mathrm{i}=t}^{\mathrm{L}-1}\left(\frac{\mathrm{P}_{\mathrm{i}}}{\mathrm{P}^{\mathrm{B}}}\right)}{\mathrm{q}-1}, \mathrm{P}^{\mathrm{B}}=\sum_{\mathrm{i}=\mathrm{t}}^{\mathrm{L}-1} \mathrm{P}_{\mathrm{i}} .
\end{aligned}
$$

The information measures between the two classes (object and background) are maximized. When $\mathrm{S}_{\mathrm{q}}{ }^{\mathrm{A}}(\mathrm{t})$ is maximized, the luminance level $t$ is considered to be the optimum threshold value. This can be achieved by a cheap computational effort. This Tsallis entropy criterion method can also be extended to multilevel thresholding and it is described as follows:

$$
\begin{array}{r}
\mathrm{f}(\mathrm{t})=\operatorname{argmax}\left[\mathrm{S}_{\mathrm{q}}{ }^{\mathrm{A}}(\mathrm{t})+\mathrm{S}_{\mathrm{q}}{ }^{\mathrm{B}}(\mathrm{t})+\mathrm{S}_{\mathrm{q}}{ }^{\mathrm{C}}(\mathrm{t})+\ldots+\mathrm{S}_{\mathrm{q}}{ }^{\mathrm{m}}(\mathrm{t})+\right. \\
\left.(1-\mathrm{q}) \cdot \mathrm{S}_{\mathrm{q}}{ }^{\mathrm{A}}(\mathrm{t}) \cdot \mathrm{S}_{\mathrm{q}}{ }^{\mathrm{B}}(\mathrm{t}) \cdot \mathrm{S}_{\mathrm{q}}{ }^{\mathrm{C}}(\mathrm{t}) \ldots . \mathrm{S}_{\mathrm{q}}{ }^{\mathrm{m}}(\mathrm{t})\right]
\end{array}
$$

where

$$
\begin{aligned}
& \mathrm{S}_{\mathrm{q}}^{\mathrm{A}}(\mathrm{t})=\frac{1-\sum_{\mathrm{i}=0}^{1}\left(\frac{\mathrm{i}}{\mathrm{P}^{\mathrm{A}}}\right)}{\mathrm{q}-1}, \mathrm{P}^{\mathrm{A}}=\sum_{\mathrm{i}=0}^{\mathrm{t}-1} \mathrm{P}_{\mathrm{i}} \\
& \mathrm{S}_{\mathrm{q}}^{\mathrm{B}}(\mathrm{t})=\frac{1-\sum_{\mathrm{i}=\mathrm{t}}^{2}{ }_{1}^{\mathrm{t}}\left(\frac{\mathrm{i}}{\mathrm{P}^{\mathrm{B}}}\right)}{\mathrm{q}-1}, \mathrm{P}^{\mathrm{B}}=\sum_{\mathrm{i}=\mathrm{t}}^{\sum_{1}} \mathrm{P}_{\mathrm{i}} \\
& S_{q}^{C}(t)=\frac{1-\sum_{i=t}^{3}\left(\frac{P_{i}}{C_{2}}\right)}{q-1}, P^{C}=\sum_{i=t}^{t^{3}} P_{i} \cdots
\end{aligned}
$$

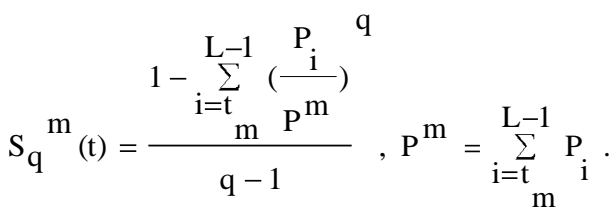

The aim of this proposed PSO algorithm is to maximize the Tsallis objective function using equations (2).

\section{OVERVIEW OF PSO}

PSO is a stochastic global optimization technique which uses swarming behaviors observed in flock of birds, school of fishes or swarm of bees, in which the intelligence is emerged. It was developed in 1995, by James Kennedy and Russell Eberhart and uses a number of particles that constitute a swarm moving around in an $\mathrm{N}$ - dimensional search space looking for the best solution. Each particle keeps track of its coordinates in the solution space which are associated with the best solution that has achieved so far by that particle is called as personal best position(pbest) and the another best value obtained so far by any particle in the neighborhood of that particle is called as global best position (gbest).

Each particle tries to modify its position using the following information.

- The current positions

- The current velocities 
- The distance between the current position and pbest

- The distance between the current position and gbest

\subsection{Advantages of PSO}

$>$ PSO is easy to implement and only few parameters have to be adjusted.

D Unlike the GA, PSO has no evolution operators such as crossover and mutation.

$>$ In GAs, chromosomes share information so that the whole population moves like one group, but in PSO, only global best particle (gbest) gives out information to the others. It is more robust than GAs.

$>$ PSO can be more efficient than GAs; that is, PSO often finds the solution with fewer objective function evaluations than that required by GAs.

D Unlike GAs and other heuristic algorithms, PSO has the flexibility to control the balance between global and local exploration of the search space.

\subsection{PSO algorithm}

Let $\mathrm{X}$ and $\mathrm{V}$ denote the particle's position and its corresponding velocity in search space respectively. At iteration $\mathrm{K}$, each particle $\mathrm{i}$ has its position defined by $\mathrm{X}_{\mathrm{i}} \mathrm{K}^{\mathrm{N}}=\left[\mathrm{X}_{\mathrm{i}, 1}, \mathrm{X}_{\mathrm{i}, 2} \ldots \mathrm{X}_{\mathrm{i}, \mathrm{N}}\right]$ and a velocity is defined as $\quad \mathrm{V}_{\mathrm{i}}^{\mathrm{K}}=\left[\mathrm{V}_{\mathrm{i}, 1}, \quad \mathrm{~V}_{\mathrm{i}, 2} \ldots \ldots \mathrm{V}_{\mathrm{i}, \mathrm{N}}\right]$ in search space N. Velocity and position of each particle in the next iteration can be calculated as

$$
\begin{aligned}
& \mathrm{V}_{\mathrm{i}, \mathrm{n}}{ }^{\mathrm{k}+1}=\mathrm{W} \times \mathrm{V}_{\mathrm{i}, \mathrm{n}}{ }^{\mathrm{k}}+\mathrm{C}_{1} \times \operatorname{rand}_{1} \times\left(\text { pbest }_{\mathrm{i}, \mathrm{n}}-\mathrm{X}_{\mathrm{i}, \mathrm{n}}{ }^{\mathrm{k}}\right)+\mathrm{C}_{2} \times \text { rand }_{2} \\
& \times\left(\text { gbest }_{\mathrm{n}}-\mathrm{X} \mathrm{i}_{\mathrm{n}}{ }^{\mathrm{k}}\right) \\
& \mathrm{i}=1,2 \ldots \ldots \ldots \mathrm{p} \\
& \mathrm{n}=1,2 \ldots \ldots \ldots \mathrm{m} \\
& \begin{aligned}
X_{i, n}{ }^{k+1} & =X_{i, n}{ }^{k}+V_{i, n}{ }^{k+1} & & \text { if } X_{\min , i, n} \leq X_{i}{ }^{k+1} \leq X_{\max i, n} \\
& =X_{\min i, n} & & \text { if } X_{i, n+1}{ }^{k+1} X_{\min i, n} \\
& =X_{\max i, n} & & \text { if } X_{i, n}{ }_{k+1}>X_{\max i, n}
\end{aligned}
\end{aligned}
$$

The inertia weight $\mathrm{W}$ is an important factor for the PSO's convergence. It is used to control the impact of previous history of velocities on the current velocity. A large inertia weight factor facilitates global exploration (i.e., searching of new area) while small weight factor facilitates local exploration. Therefore, it is better to choose large weight factor for initial iterations and gradually reduce weight factor in successive iterations. This can be done by using

$$
\mathrm{W}=\mathrm{W}_{\text {max }}-\left(\mathrm{W}_{\text {max }}-\mathrm{W}_{\text {min }}\right) \times \text { Iter } / \text { Iter }_{\text {max }}
$$

Where $\mathrm{W}_{\max }$ and $\mathrm{W}_{\min }$ are initial and final weight respectively, Iter is current iteration number and Iter $\max _{\text {ax }}$ is maximum iteration number.

Acceleration constant $\mathrm{C}_{1}$ called cognitive parameter pulls each particle towards local best position whereas constant $\mathrm{C}_{2}$ called social parameter pulls the particle towards global best position.
The particle position is modified by equation (4). The process is repeated until stopping criterion is reached.

\section{IMPLEMENTATION OF PSO FOR MULTILEVEL THRESHOLDING PROBLEM}

This paper presents a quick solution to the multilevel image thresholding problems using the PSO algorithm. The number of threshold levels is the dimension of the problem. For example, if there are ' $\mathrm{m}$ ' threshold levels, the ith particle is represented as follows:

$$
\mathrm{X}_{\mathrm{i}}=\left(\mathrm{X}_{\mathrm{i} 1}, \mathrm{X}_{\mathrm{i} 2}, \ldots \ldots \ldots, \mathrm{X}_{\mathrm{im}}\right)
$$

Its implementation consists of the following steps.

Step 1. Initialization of the swarm: For a population size $\mathrm{p}$, the particles are randomly generated between the minimum and the maximum limits of the threshold values.

Step 2. Evaluation of the objective function: The objective function values of the particles are evaluated using the objective functions given by equation (2).

Step 3. Initialization of pbest and gbest: The objective values obtained above for the initial particles of the swarm are set as the initial pbest values of the particles. The best value among all the pbest values is identified as gbest.

Step 4. Evaluation of velocity: The new velocity for each particle is computed using equation (3).

Step 5. Update the swarm: The particle position is updated using equation (4). The values of the objective function are calculated for the updated positions of the particles. If the new value is better than the previous pbest, the new value is set to pbest. Similarly, gbest value is also updated as the best pbest.

Step 6. Stopping criteria: If the stopping criteria are met, the positions of particles represented by gbest are the optimal threshold values. Otherwise, the procedure is repeated from step 4 .

\section{TEST RESULTS AND DISCUSSIONS}

The performance of the proposed particle swarm optimization (PSO) based multilevel thresholding algorithm is evaluated. Some experiments with several test images are presented to illustrate the key features of the proposed method in the determination of the optimal threshold values and its efficiency for thresholding computation. Comparisons are performed with results provided by GA.

Six well known images named Lenna, Pepper, Baboon, Hunter, Cameraman and Airplane with 256 grey levels are used. All the images are of size $512 \times 512$ except the pepper image which is of size $256 \times 256$. These images are gathered in Fig.1 with their respective histograms. The experiments are performed on a Pentium IV, core to duo, 3 GHZ PC. 

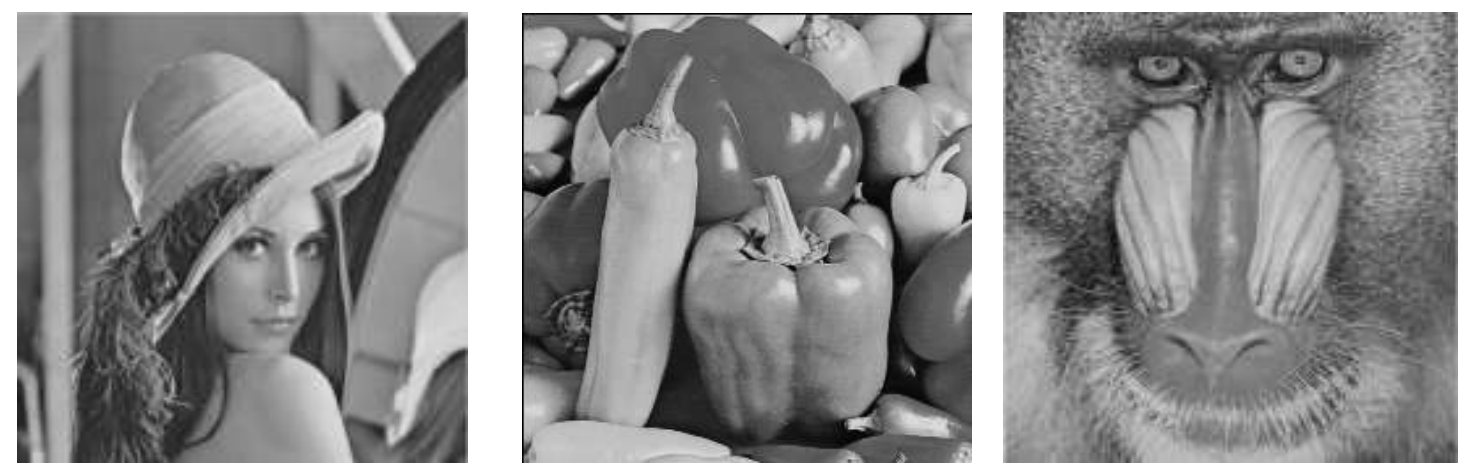

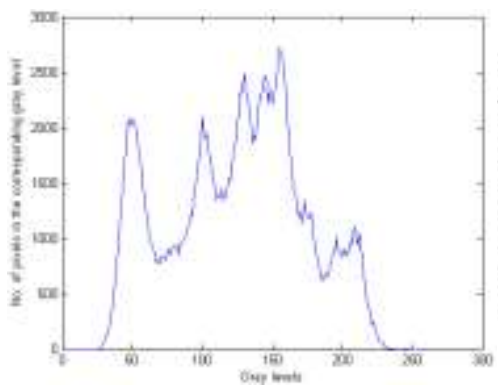

(a)
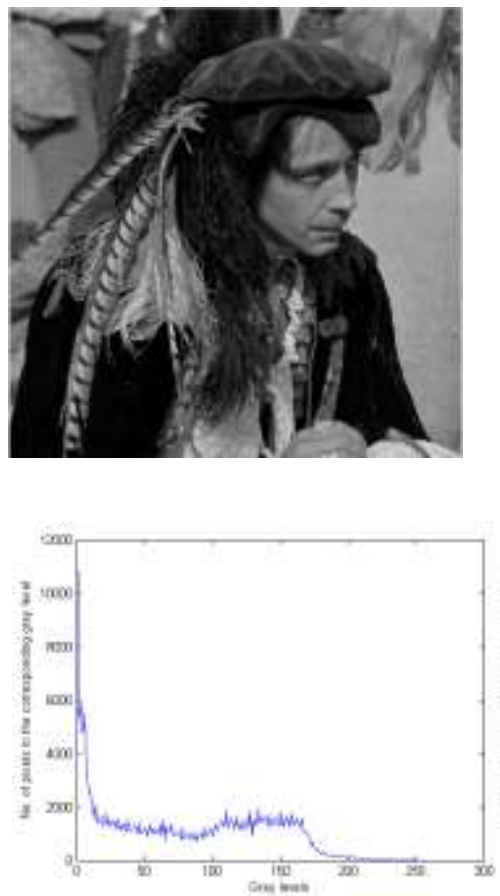

(d)

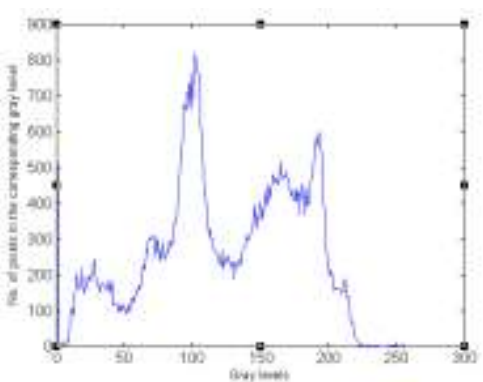

(b)
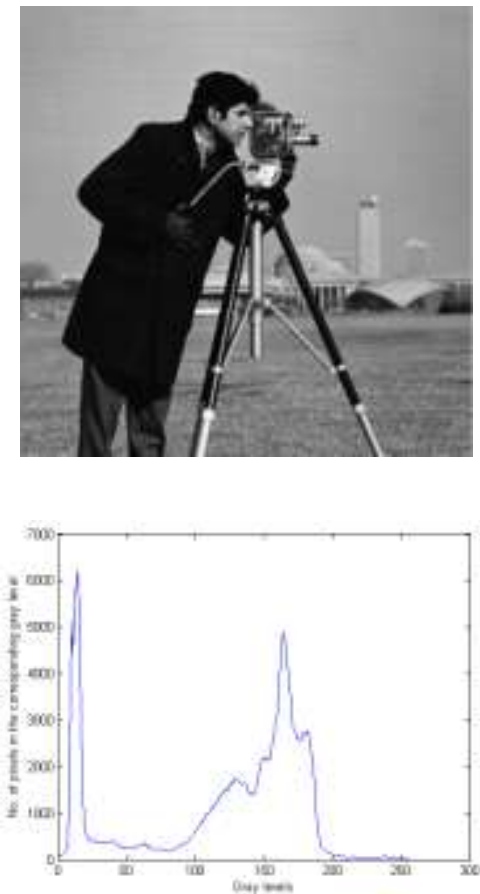

(e)
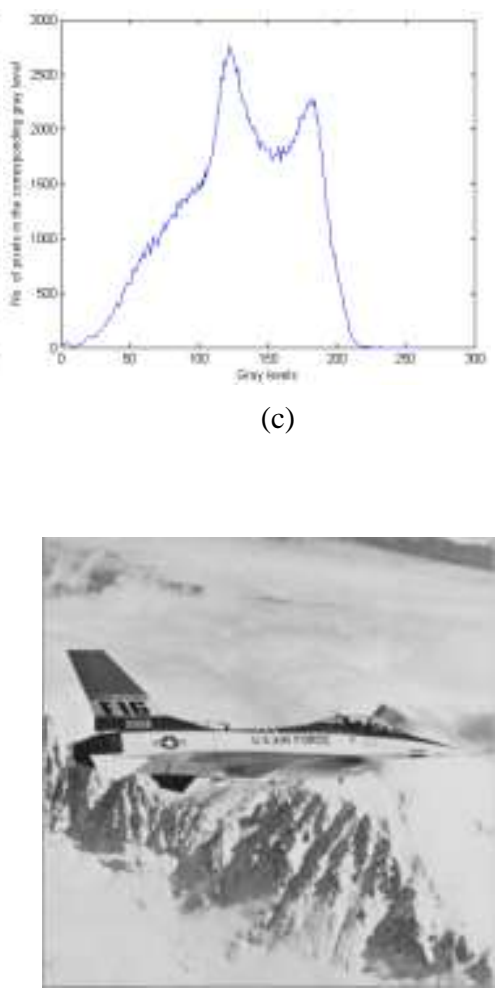

(c)

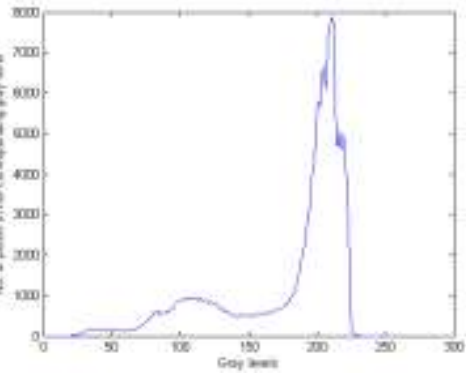

(f)

Figure 1. Test Images and their histograms

(a) Lena, (b) Pepper, (c) Baboon, (d) Hunter, (e) Cameraman, (f) Airplane 
Table 1 Optimal threshold values and their objective value obtained by PSO and GA methods

\begin{tabular}{|c|c|l|l|c|c|}
\hline \multirow{2}{*}{ Test Images } & \multirow{2}{*}{$\mathrm{m}$} & \multicolumn{2}{|c|}{ Optimal threshold values } & \multicolumn{2}{c|}{ Objective values } \\
\cline { 3 - 6 } & & \multicolumn{1}{|c|}{ PSO } & \multicolumn{1}{|c|}{ GA } & PSO & GA \\
\hline \multirow{5}{*}{ LENNA } & 2 & 120,164 & 120,164 & 0.8889 & 0.8889 \\
\cline { 2 - 6 } & 3 & $110,149,187$ & $98,159,181$ & 1.296268 & 1.296247 \\
\cline { 2 - 6 } & 4 & $85,118,164,200$ & $86,120,151,205$ & 1.654255 & 1.654208 \\
\cline { 2 - 6 } & 5 & $86,117,142,166,196$ & $95,130,152,173,200$ & 1.995773 & 1.995717 \\
\hline \multirow{5}{*}{ PEPPER } & 2 & 82,154 & 82,154 & 0.8889 & 0.8889 \\
\cline { 2 - 6 } & 3 & $93,133,179$ & $75,103,182$ & 1.296274 & 1.296262 \\
\cline { 2 - 6 } & 4 & $73,121,141,176$ & $73,109,141,193$ & 1.654248 & 1.654225 \\
\cline { 2 - 6 } & 5 & $78,111,141,169,198$ & $78,105,139,168,200$ & 1.995766 & 1.995739 \\
\hline \multirow{5}{*}{ BABOON } & 2 & 91,147 & 91,147 & 0.8889 & 0.8889 \\
\cline { 2 - 6 } & 3 & $108,155,181$ & $111,136,193$ & 1.296274 & 1.296202 \\
\cline { 2 - 6 } & 4 & $62,115,144,174$ & $94,125,152,177$ & 1.654262 & 1.654241 \\
\cline { 2 - 6 } & 5 & $84,110,132,153,175$ & $90,116,139,159,180$ & 1.995737 & 1.995708 \\
\hline \multirow{5}{*}{ HUNTER } & 2 & 94,137 & 94,137 & 0.8889 & 0.8889 \\
\cline { 2 - 6 } & 3 & $83,143,174$ & $87,147,173$ & 1.296267 & 1.296227 \\
\cline { 2 - 6 } & 4 & $78,109,143,187$ & $90,119,150,191$ & 1.654255 & 1.654240 \\
\cline { 2 - 6 } & 5 & $70,103,139,174,198$ & $79,114,144,174,198$ & 1.995720 & 1.995713 \\
\hline \multirow{5}{*}{ CAMERAMAN } & 2 & 120,154 & 120,154 & 0.8889 & 0.8889 \\
\cline { 2 - 6 } & 3 & $78,121,173$ & $81,143,170$ & 1.296180 & 1.296141 \\
\cline { 2 - 6 } & 4 & $82,122,154,201$ & $76,116,148,202$ & 1.654183 & 1.654177 \\
\cline { 2 - 6 } & 5 & $78,110,133,159,199$ & $88,118,143,169,205$ & 1.995669 & 1.995663 \\
\hline \multirow{5}{*}{ AIRPLANE } & 2 & 72,153 & 72,153 & 0.8889 & 0.8889 \\
\cline { 2 - 6 } & 3 & $98,134,192$ & $89,148,172$ & 1.296204 & 1.296180 \\
\cline { 2 - 6 } & 4 & $85,117,153,180$ & $79,111,153,173$ & 1.654243 \\
\cline { 2 - 6 } & 5 & $75,107,134,157,185$ & $73,98,131,162,192$ & & \\
\end{tabular}



(a)

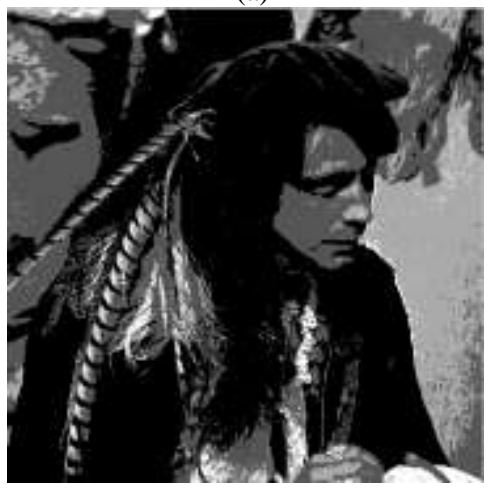

(d)

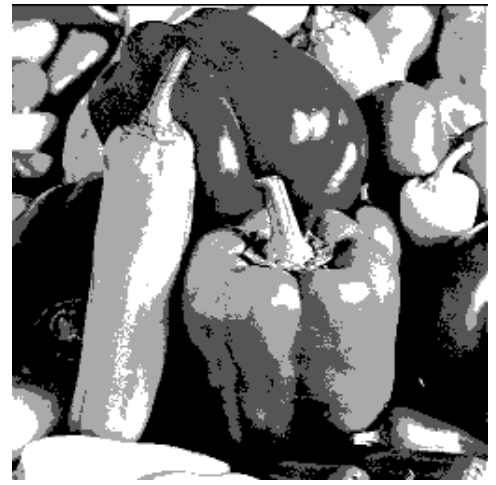

(b)

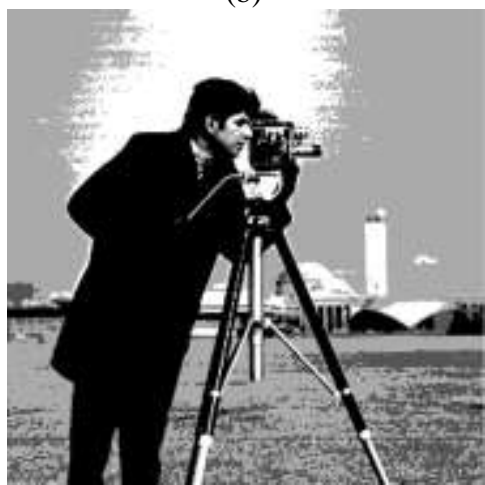

(e)

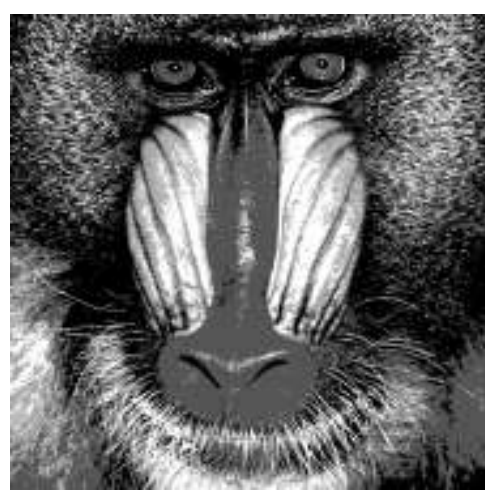

(c)

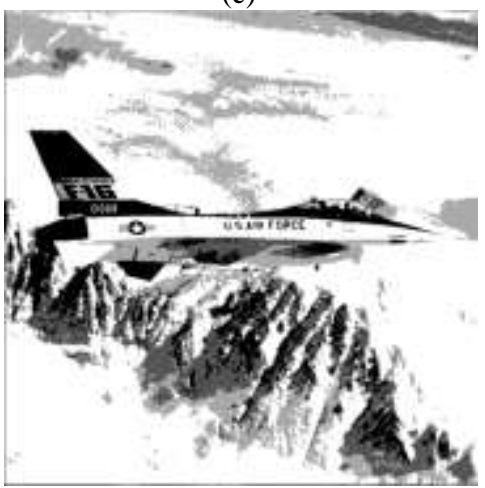

(f)

Figure 2. 3-level thresholding images

(a) Lena, (b) Pepper, (c) Baboon, (d) Hunter, (e) Cameraman, (f) Airplane 


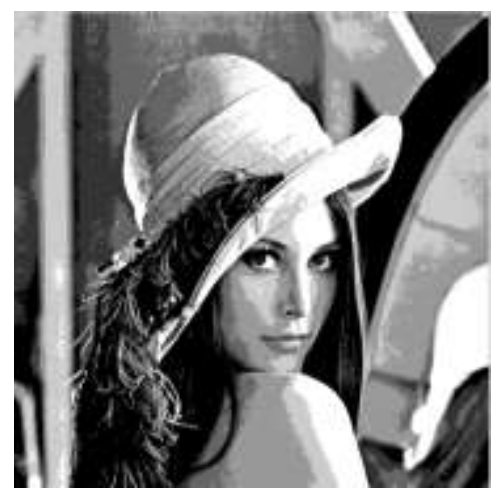

(a)

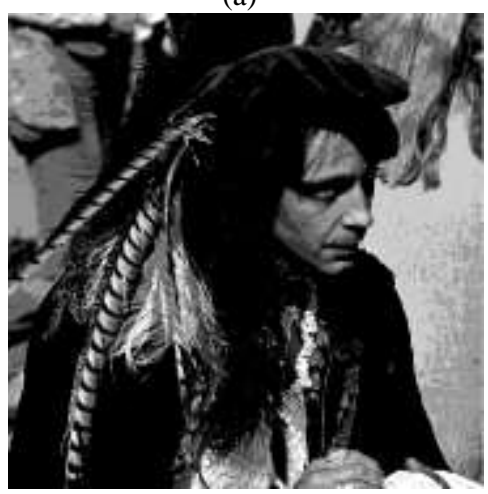

(d)

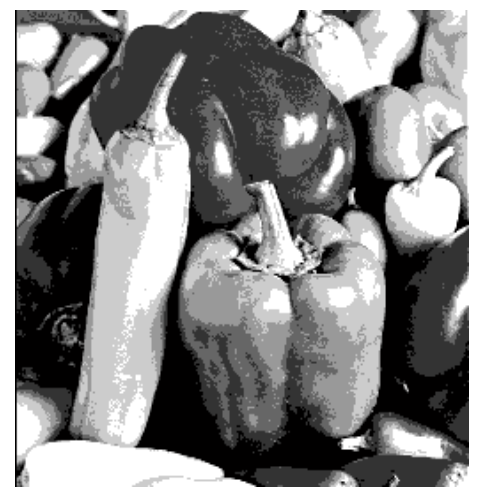

(b)

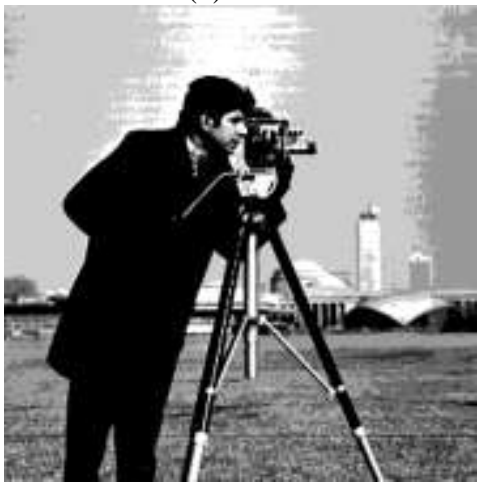

(e)



(c)

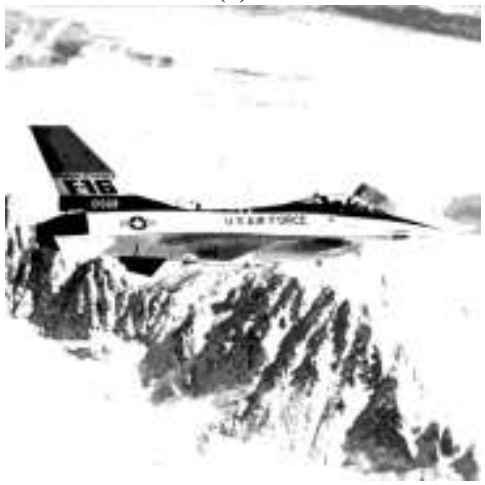

(f)

Figure 3. 5-level thresholding images (a) Lena, (b) Pepper, (c) Baboon, (d) Hunter, (e) Cameraman, (f) Airplane

Table 2 PSNR value, Computational time and standard deviation obtained by PSO and GA methods

\begin{tabular}{|c|c|c|c|c|c|c|c|}
\hline \multirow{2}{*}{ Test Images } & \multirow{2}{*}{$\mathrm{m}$} & \multicolumn{2}{|c|}{ PSNR (db) } & \multicolumn{2}{|c|}{ Standard Deviation } & \multicolumn{2}{|c|}{ CPU Time (sec) } \\
\hline & & PSO & GA & PSO & GA & PSO & GA \\
\hline \multirow{4}{*}{ LENNA } & 2 & 15.2419 & 15.2419 & 0.0000 & 0.0000 & 3.6810 & 3.9219 \\
\hline & 3 & 17.1425 & 16.9455 & $2.5418 \mathrm{e}-006$ & $3.8999 \mathrm{e}-006$ & 4.0357 & 4.3906 \\
\hline & 4 & 19.4324 & 19.0207 & $1.3306 \mathrm{e}-005$ & $1.9104 \mathrm{e}-005$ & 4.7523 & 4.8438 \\
\hline & 5 & 20.5637 & 19.8703 & $1.6797 \mathrm{e}-005$ & $2.7208 \mathrm{e}-005$ & 4.9900 & 5.2854 \\
\hline \multirow{4}{*}{ PEPPER } & 2 & 12.9108 & 12.9108 & 0.0000 & 0.0000 & 3.5394 & 3.9844 \\
\hline & 3 & 16.0269 & 15.5628 & $7.3578 \mathrm{e}-006$ & $2.0199 \mathrm{e}-005$ & 3.5473 & 3.9919 \\
\hline & 4 & 16.7109 & 16.3735 & $7.0094 \mathrm{e}-006$ & $1.7406 \mathrm{e}-005$ & 4.4063 & 5.0938 \\
\hline & 5 & 20.2089 & 19.7642 & $6.3010 \mathrm{e}-006$ & $1.1678 \mathrm{e}-005$ & 4.8484 & 5.2314 \\
\hline \multirow{4}{*}{ BABOON } & 2 & 13.1404 & 13.1404 & 0.0000 & 0.0000 & 3.5021 & 3.8906 \\
\hline & 3 & 17.0809 & 16.7728 & $9.3397 \mathrm{e}-006$ & $1.2993 \mathrm{e}-005$ & 4.2591 & 4.4422 \\
\hline & 4 & 17.1462 & 17.1583 & $7.2225 \mathrm{e}-006$ & $1.3714 \mathrm{e}-005$ & 4.3365 & 4.5156 \\
\hline & 5 & 18.2718 & 17.2903 & $1.1321 \mathrm{e}-005$ & $1.8993 \mathrm{e}-005$ & 5.4188 & 5.8281 \\
\hline \multirow{4}{*}{ HUNTER } & 2 & 11.3848 & 11.3848 & 0.0000 & 0.0000 & 3.6970 & 3.9797 \\
\hline & 3 & 14.5135 & 14.0724 & $1.8965 \mathrm{e}-006$ & $1.0060 \mathrm{e}-005$ & 4.0130 & 4.3906 \\
\hline & 4 & 15.4496 & 14.1926 & $4.2172 \mathrm{e}-006$ & $1.0886 \mathrm{e}-005$ & 4.6875 & 4.7031 \\
\hline & 5 & 16.6426 & 15.6197 & $1.2255 \mathrm{e}-005$ & $9.3619 \mathrm{e}-005$ & 5.0009 & 5.4688 \\
\hline \multirow{4}{*}{ CAMERAMAN } & 2 & 10.6258 & 10.6258 & 0.0000 & 0.0000 & 3.0021 & 3.6482 \\
\hline & 3 & 14.9951 & 14.5900 & $5.4543 \mathrm{e}-006$ & $8.4892 \mathrm{e}-006$ & 3.7658 & 4.3906 \\
\hline & 4 & 15.9187 & 14.9756 & $7.5181 \mathrm{e}-006$ & $1.1024 \mathrm{e}-005$ & 4.6188 & 4.8594 \\
\hline & 5 & 17.2393 & 16.6026 & $1.0319 \mathrm{e}-005$ & $7.7199 \mathrm{e}-005$ & 5.1343 & 5.6026 \\
\hline \multirow{4}{*}{ AIRPLANE } & 2 & 13.7290 & 13.7290 & 0.0000 & 0.0000 & 3.3159 & 3.8921 \\
\hline & 3 & 15.5913 & 14.6681 & $3.1114 \mathrm{e}-006$ & $6.9412 \mathrm{e}-006$ & 3.7625 & 4.1358 \\
\hline & 4 & 15.6294 & 14.9701 & $2.6305 \mathrm{e}-006$ & $9.2004 \mathrm{e}-006$ & 4.8750 & 5.2656 \\
\hline & 5 & 17.6077 & 16.1579 & $3.3007 \mathrm{e}-006$ & $6.3861 \mathrm{e}-006$ & 5.2813 & 5.6077 \\
\hline
\end{tabular}


The proposed multilevel thresholding technique using PSO is implemented with the following parameters.

Swam Size: 20,

No. of Iterations: 100 ,

$\mathrm{W}_{\text {max }}, \mathrm{W}_{\text {min: }} 0.4,0.1$, and

$\mathrm{C}_{1}, \mathrm{C}_{2}: 2$.

In Tsallis objective function, the parameter $\mathrm{q}$ is chosen as 4 .

Table 1 shows the number of thresholds, the optimal threshold values and the objective values achieved by the proposed method when an entropic index $\mathrm{q}$ is chosen as 4. Both PSO and GA methods use the Eq. (2) as objective function to decide whether the number of thresholds has reached the optimal value or not. The higher value of objective function results in better segmentation. From Table 1, it is observed that the proposed PSO method obtains higher objective value than GA.

Fig. 2 and 3 show the thresholded images for $m=3$ and 5 respectively. The quality of the segmentation is better when $\mathrm{m}=5$ is chosen for all the images. The quality of the thresholded images can also be evaluated through Peak Signal to Noise Ratio (PSNR) measure.

$$
\mathrm{PSNR}=20 \log _{10}\left(\frac{255}{\mathrm{RMSE}}\right)
$$

where

$$
\operatorname{RMSE}=\sqrt{\frac{1}{M N} \sum_{i=1}^{M} \sum_{j=1}^{N}[I(i, j)-\hat{I}(i, j)]^{2}}
$$

Table 2 shows the PSNR value obtained by the PSO and GA methods. The higher value of PSNR means that the quality of the thresholded image is better. For all the images, the performance of the proposed method is better than the GA, since their objective value and PSNR measure are higher. In the view point of the computation time, the proposed method is faster than the GA. It is shown in Table 2. The CPU time increases with the number of thresholds.

As all the optimization algorithms are stochastic and random searching one, the results of experiments are not absolutely the same in each run of the algorithm. Hence, it is necessary to analyze the stability of all the algorithms. This comparison is utilized to find which algorithm is more stable than others. The stability is expressed in the form of standard deviation as

$$
\operatorname{std}=\sqrt{\sum_{\mathrm{i}=1}^{\mathrm{k}} \frac{\left(\sigma_{\mathrm{i}}-\mu\right)^{2}}{\mathrm{k}}}
$$

where std represents the standard deviation, $\mathrm{k}$ is the number of runs of each algorithm $(\mathrm{k}=100), \sigma_{\mathrm{i}}$ is the best objective value obtained by the ith run of the algorithm, $\mu$ represents the mean value of $\sigma$. The standard deviation values of each algorithm are furnished in Table 2. From the results, the standard deviation value of PSO algorithm is lesser than GA which illustrates the stability of the proposed PSO algorithm.

\section{CONCLUSION}

Nonextensive entropy image thresholding is a powerful technique for image segmentation. In this paper, a new multilevel thresholding method based on Particle Swarm Optimization (PSO) is proposed which enables in determining the optimal threshold values by maximizing Tsallis objective function. Experiments with several standard test images have proved the robustness of the proposed method, in view of the accuracy of image segmentation, evaluated through the PSNR measure and the objective function. Comparison with the GA method showed that the proposed method runs faster, and more stable.

\section{REFERENCES}

[1] M. Sezgin, B. Sankur. "Survey over image thresholding techniques and quantitative performance evaluation", Journal of Electronic Imaging, 13 (1) (2004), 146-165.

[2] A.D. Brink, "Minimum spatial entropy threshold selection", IEE Proceedings on Vision Image and Signal Processing 142 (1995), 128-132.

[3] J.N. Kapur, P.K. Sahoo, A.K.C.Wong, “A new method for gray-level picture thresholding using the entropy of the histogram," Computer Vision, Graphics and Image Processing 29 (1985), 273-285.

[4] N. Otsu, "A threshold selection method from gray level histograms. IEEE Transactions on Systems, Man and Cybernetics SMC-9 (1979), 62-66.

[5] T. Cover, J. Thomas, "Elements of information theory", John Wiley and Sons, Inc (1991).

[6] T. Pun, "A new method for gray level picture thresholding using the entropy of the histogram", Signal Processing 2 (1980), 223-237.

[7] T. Pun, "Entropic thresholding: A new approach", Comput. Graph. Image Process. 16 (1981), 210-239.

[8] C. Tsallis, In: S. Abe, Y. Okamoto (Eds), "Non-extensive statistical Mechanics and its applications," Series Lecture Notes in Physics, Springer, Berlin (2001).

[9] P. S. T. Liao, S. Chen, and P. C. Chung, "A fast algorithm for multilevel thresholding", Journal of Information science and Engineering 17 (2001) 713-727.

[10] K. C. Lin, "Fast image thresholding by finding zero(s) of the first derivative of between class variance", Machine Vision and Applications 13 (2003), 254-262.

[11] Peng-Yeng Yin and Ling-Hwei Chen, "A fast iterative scheme for multilevel thresholding methods," Signal Processing 60 (3) (1997), 305-313.

P. Y. Yin, "A fast scheme for optimal thresholding using genetic algorithms", Signal processing 72 (1999), 85-95.

C. C. Lai, D. C. Tseng, "A hybrid approach using Gaussian smoothing and genetic algorithm for multilevel thresholding", International Journal of Hybrid Intelligent Systems 1(3) (2004), 143-152.

[14] D. B. Fogel, "Evolutionary computation: Toward a new philosophy of machine intelligence, Second edition, Piscataway, NJ: IEEE Press, 2000. 
[15] J. Kennedy, and R. Eberhart, "Particle Swarm Optimization", Proceedings of the IEEE conference on neural networks ICNN'95, Perth, Australia 4 (1995), 1942-1948.

[16] Yi-Tung Kao, Erwie Zahara and I-Wei Kao, " A hybridized approach to data clustering," Expert Systems with [18] Applications 34 (2008), 1754-1762.
[17] Zne-Jung Lee, Shin-Wei Lin, Shun-Feng Su and Chun-Yen Lin, " A hybrid watermarking technique applied to digital images”, Expert Systems with Applications 8 (2008), 789808.

18] Chun-Chieh Tseng, Jer-Guang Hsieh and Jyh-Horng Jeng, “ Fractal image compression using visual-based particle swarm optimization," Image and Vision Computing 26 (8) (2008), 1154-1162. 